Original Research Paper

\title{
Cross-Section Optimization of Sandwich-Type Cylindrical Wind Turbine Towers
}

\author{
Stylianos M. Vernardos and Charis J. Gantes \\ School of Civil Engineering, National Technical University of Athens, Athens, Greece
}

Article history

Received: 23-07-2015

Revised: $30-07-2015$

Accepted: 31-07-2015

Corresponding Author: Stylianos M. Vernardos

School of Civil Engineering,

National Technical University of

Athens, Athens, Greece

Email: s.vernardos@gmail.com

\begin{abstract}
As wind energy is aggressively expanding within the alternative sources' part of modern world's energy pie, the need of addressing various obstacles that limit further wind utilization becomes all the more urgent. From a structural engineering point of view, surmounting the tower-base diameter limitation, dictated by the current transportation capabilities, is one of the industry's primary issues. The concept presented in this study, as a means to address this issue, is the implementation of a composite, sandwichtype tower section instead of today's predominant, conventional steel-only tubular section. The proposed section consists of two steel faces and a core of some lightweight material, binding and keeping them at a specified distance, thus providing significantly improved mechanical properties with minimal additional weight and cost. As an initial step towards a feasibility investigation of the proposed section, the analytical expressions that govern its behavior under either axial or bending loads are formulated in the current study. The results are verified by comparison with those obtained from a specialized composite section software, as well as a general purpose finite element software. The analytical solution is then employed to carry out extensive parametric analyses, involving a wide range of material qualities and layer thicknesses, leading to the optimization of the section, in terms of either elastic bending strength or initial stiffness. Finally, the determination of the optimal section-properties, which provide the most efficient solution regarding both criteria, is addressed, resulting in a preliminary design tool for sandwich-type, wind-turbine-tower sections.
\end{abstract}

Keywords: Wind Turbine Tower, Composite Section, Optimization, Cylindrical Shell

\section{Introduction}

The ambitious goals set by governmental organizations worldwide, regarding the contribution of wind energy to global energy supply, are hindered by several, practical difficulties. As wind turbines reach greater heights, in order to take advantage of higher wind velocities, the tower becomes more flexible, leading to increased lateral deformations, possible danger of resonance with the electro-mechanical parts of the turbine and increased fatigue problems at the connections (Burton et al., 2001; Hau, 2006; Basu, 2010). From a mechanics point of view, the obvious way to deal with this is to use a larger tower diameter, particularly at lower heights, in order to increase stiffness and strength. Increasing the thickness instead of the diameter is a lot less effective, as it not only imposes heavier material requirements and unproportionally higher costs, but it also introduces higher residual stresses during cold curving of the steel plates into conical shells, thus reducing the elastic response range of the tower under service loads (Burton et al., 2001; DNV, 2002; Hau, 2006; Twele et al., 2012). However, increase of the tower diameter is bounded by the available means of transportation of individual parts, particularly on mountain tops where wind parks are usually located. In order to remain transferable through narrow roads and under overhead obstructions, tower sections cannot generally exceed a maximum diameter of $4.5 \mathrm{~m}$, which consequently limits total tower heights to approximately 110 m (Burton et al., 2001; DNV, 2002; Hau, 2006; Twele et al., 2012; NREL, 2014).

To address this issue, several alternatives for wind turbine tower design have been proposed, none of which 
comes without more or less serious drawbacks. Lattice, concrete and hybrid steel-concrete towers are some examples (Twele et al., 2012).

The concept investigated in the current research endeavor is, instead, that of a composite tower section consisting of two steel faces and a core made of some lightweight material, thus resembling a sandwich form. The advantage of such a solution is increased stiffness and strength with relatively small disadvantages in terms of mass and cost addition. Although the sandwich concept has been implemented in various cases and is not a novel one whatsoever, the idea of utilizing it as a wind turbine tower section is still at research level and is only mentioned in the literature in limited works. In their preliminary investigation, Schaumann and Keindorf (2008) compared three different sandwich shells with solid steel ones ("monocoque") on the basis of ultimate limit state calculations and concluded that sandwich shells are more efficient with respect to buckling. Lim et al. (2013) worked on the geometrical optimization of a sandwich-section tower, manufactured by the filament winding method using glass/epoxy materials, under wind loads. They pointed out its advantages compared to conventional steel towers, in terms of stability under wind loads, as well as weight and cost efficiency. The authors of the current paper (Vernardos and Gantes, 2014) investigated the feasibility of a sandwich section, consisting of two steel faces and concrete core, under bending load and attempted a preliminary optimization regarding layer thicknesses and material qualities.

Regarding material selection, modern materials such as various forms of honeycombs or metal foams appear to be an ideal choice for the core of a sandwich section due to their high stiffness and low specific weight (Simančík, 2001; Banhart, 2001). However, besides the rapid development in the field, their manufacturing costs are still retarding their widespread use (Simančík, 2001). On this basis, the use of a traditional material, such as concrete, as a means of filling the core, might be a more suitable option, considering its high ratio of beneficial physical properties to cost and is thus adopted in the current study.

\section{Analytical Solution}

\section{Sandwich Section under Axial Load}

The investigated section is qualitatively depicted in Fig. 1, while the material laws assumed for the two materials are illustrated by the diagrams of Fig. 2. The tensile strength of concrete is completely neglected at this preliminary design stage, as are any hardening and softening properties of steel and concrete, respectively. As far as the behavior of the sandwich section as a whole is concerned, full coherence is assumed and any delamination phenomena are also disregarded.

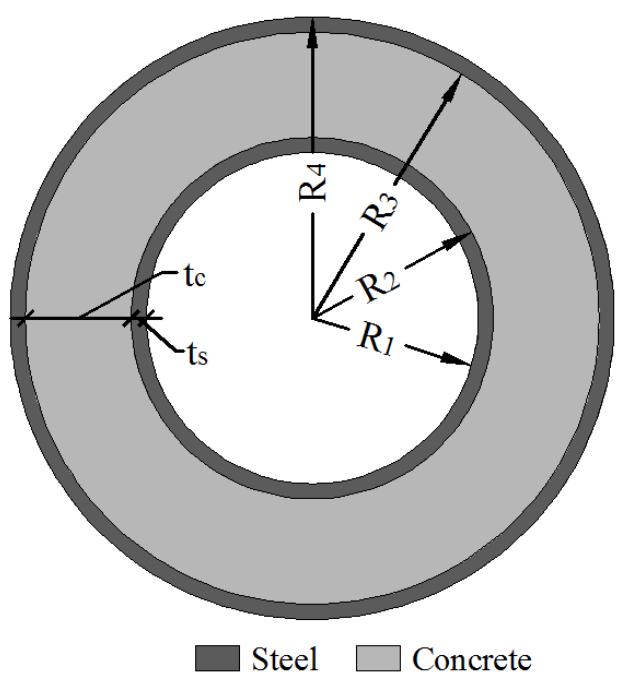

Fig. 1. Geometry of a sandwich-type section (presented thicknesses are not realistic and only outline the geometry of the section)
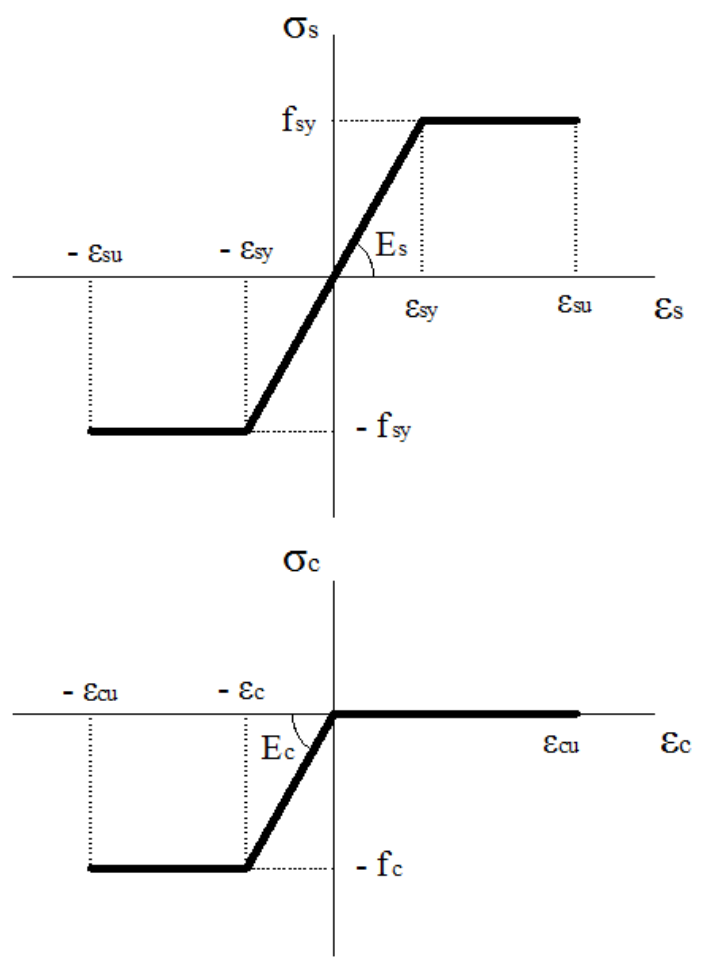

Fig. 2. Simplified stress-strain diagrams of steel (s) and concrete (c)

Equation 1 to 4 are the easily formulated analytical expressions describing the sandwich section's behavior under axial loads and are graphically presented in Fig. 3, indicatively for $E_{s}=210 \mathrm{GPa}, f_{s y}=355 \mathrm{MPa}, E_{c}=30$ $\mathrm{GPa}, f_{c}=30 \mathrm{MPa}$ and radii $R_{4}=2000 \mathrm{~mm}, R_{3}=1995$ $\mathrm{mm}, R_{2}=1895 \mathrm{~mm}, R_{1}=1890 \mathrm{~mm}$, as defined in Fig. 1: 


$$
\begin{aligned}
N_{t}= & \varepsilon \pi E_{s}\left[\left(R_{4}{ }^{2}-R_{3}^{2}\right)+\left(R_{2}^{2}-R_{1}^{2}\right)\right] \\
N_{c}= & \varepsilon \pi\left[E_{s}\left(R_{4}{ }^{2}-R_{3}{ }^{2}\right)+E_{c}\left(R_{3}{ }^{2}-R_{2}{ }^{2}\right)+\right. \\
& \left.E_{s}\left(R_{2}{ }^{2}-R_{1}{ }^{2}\right)\right] \\
N_{u, t}= & f_{s y} \pi\left[\left(R_{4}{ }^{2}-R_{3}{ }^{2}\right)+\left(R_{2}{ }^{2}-R_{1}{ }^{2}\right)\right] \\
N_{u, c}= & f_{s y} \pi\left[\left(R_{4}{ }^{2}-R_{3}{ }^{2}\right)+\left(R_{2}{ }^{2}-R_{1}{ }^{2}\right)\right]+ \\
& f_{c} \pi\left(R_{3}{ }^{2}-R_{2}{ }^{2}\right)
\end{aligned}
$$

\section{Where:}

$N_{t} \quad=$ The tensile axial load

$N_{c}=$ The compressive axial load

$\varepsilon=$ The longitudinal strain

$N_{u, t}=$ The ultimate tensile axial load

$N_{u, c}=$ The ultimate compressive axial load

$E_{s} \quad=$ The Young's modulus of steel

$E_{c}=$ The Young's modulus of concrete

$f_{s y}=$ The yield strength of steel

$f_{c}=$ The strength of concrete

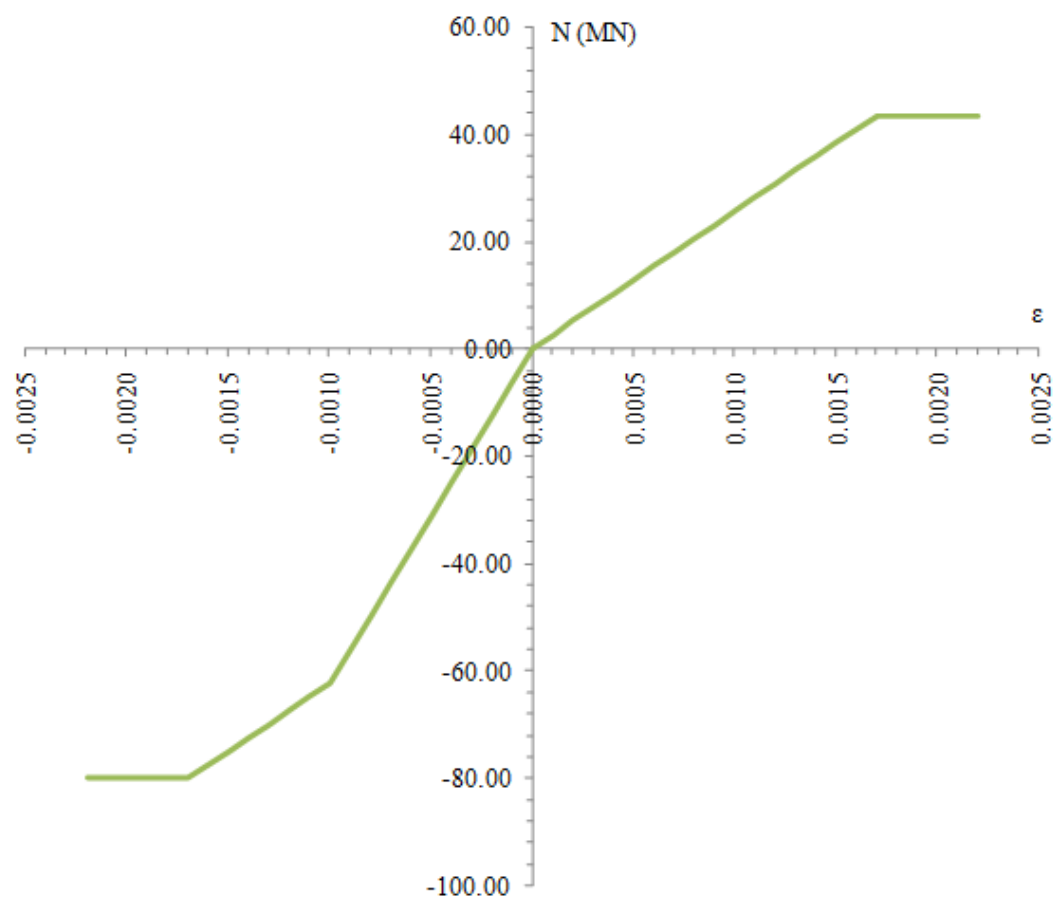

Fig. 3. Sandwich section's behavior under tension and compression for $E_{s}=210 \mathrm{GPa}, f_{s y}=355 \mathrm{MPa}, E_{c}=30 \mathrm{GPa}, f_{c}=30 \mathrm{MPa}$, $R_{4}=2000 \mathrm{~mm}, R_{3}=1995 \mathrm{~mm}, R_{2}=1895 \mathrm{~mm}, R_{1}=1890 \mathrm{~mm}$

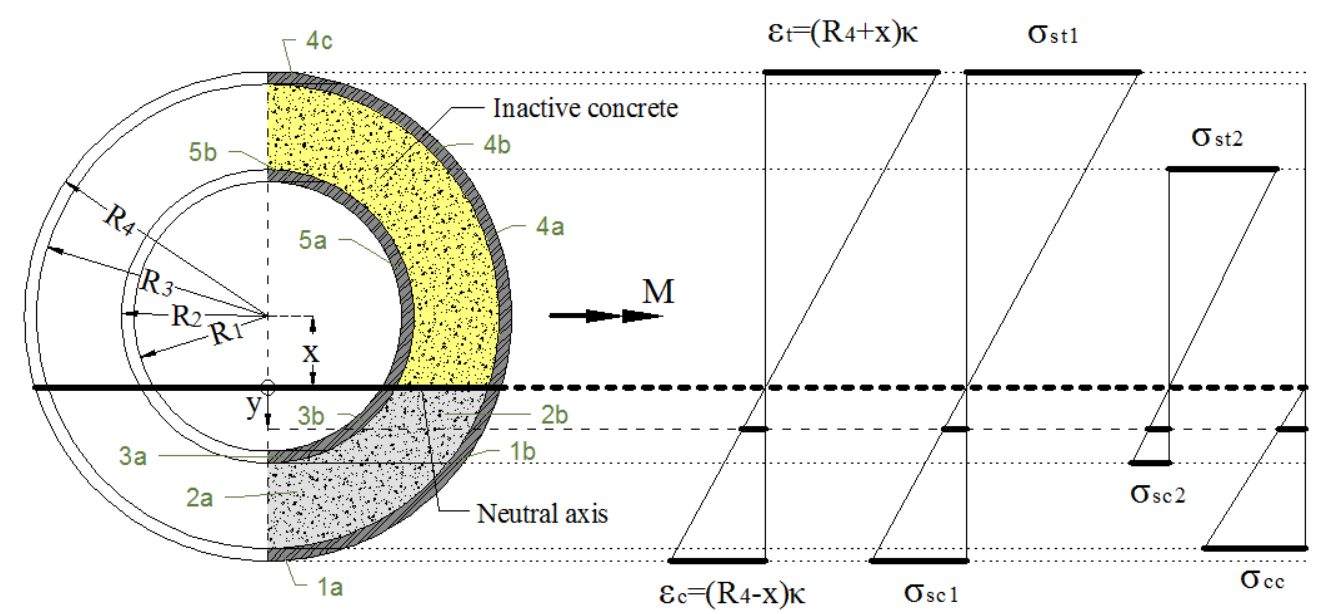

Fig. 4. Sandwich-type section under pure bending: Section fragmentation and stress-strain distributions 


\section{Sandwich Section under Bending Load}

The more complicated case of a sandwich section under bending loads was approached in this research by firstly fragmenting the section into several sectors, as shown in Fig. 4, in order for the pertinent integrations to be performed. From Equation 5 of force equilibrium, the neutral axis position can be initially determined. The computations are carried out through an iterative procedure for a random curvature value, assuming the stress and strain distributions of Fig. 4 in the elastic range. Using Equation 6, subsequently, the corresponding moment $\mathrm{M}$ exerted on the section can be calculated. Then, the initial stiffness $\overline{E I}$ of the sandwich section can be obtained from Equation 7:

$$
\begin{aligned}
& \int_{\mathrm{A}} \sigma_{x} d A=0 \Rightarrow \\
& \int_{R_{3}-x}^{R_{4}-x} E_{s} \kappa y \sqrt{R_{4}^{2}-(x+y)^{2}} d y \\
& +\int_{0}^{R_{3}-x} E_{s} \kappa y\left(\sqrt{R_{4}^{2}-(x+y)^{2}}-\sqrt{R_{3}^{2}-(x+y)^{2}}\right) d y \\
& +\int_{R_{2}-x}^{R_{3}-x} E_{c} \kappa y \sqrt{R_{3}^{2}-(x+y)^{2}} d y \\
& +\int_{0}^{R_{2}-x} E_{c} \kappa y\left(\sqrt{R_{3}^{2}-(x+y)^{2}}-\sqrt{R_{2}^{2}-(x+y)^{2}}\right) d y \\
& +\int_{R_{1}-x}^{R_{2}-x} E_{s} \kappa y \sqrt{R_{2}^{2}-(x+y)^{2}} d y \\
& +\int_{0}^{R_{1}-x} E_{s} \kappa y\left(\sqrt{R_{2}^{2}-(x+y)^{2}}-\sqrt{R_{1}^{2}-(x+y)^{2}}\right) d y \\
& -\int_{0}^{-x} E_{s} \kappa y\left(\sqrt{R_{4}^{2}-(x+y)^{2}}-\sqrt{R_{3}^{2}-(x+y)^{2}}\right) d y \\
& -\int_{-x}^{-R_{3}-x} E_{s} \kappa y\left(\sqrt{R_{4}{ }^{2}-(x+y)^{2}}-\sqrt{R_{3}^{2}-(x+y)^{2}}\right) d y \\
& -\int_{-R_{3}-x}^{-R_{4}-x} E_{s} \kappa y \sqrt{R_{4}^{2}-(x+y)^{2}} d y \\
& -\int_{0}^{-x} E_{s} \kappa y\left(\sqrt{R_{2}^{2}-(x+y)^{2}}-\sqrt{R_{1}^{2}-(x+y)^{2}}\right) d y \\
& -\int_{-x}^{-R_{1}-x} E_{s} \kappa y\left(\sqrt{R_{2}^{2}-(x+y)^{2}}-\sqrt{R_{1}^{2}-(x+y)^{2}}\right) d y \\
& -\int_{-R_{1}-x}^{-R_{2}-x} E_{s} \kappa y \sqrt{R_{2}^{2}-(x+y)^{2}} d y=0 \\
& M=\int_{A} \sigma_{x} y d A= \\
& 2\left\{\int_{R_{3}-x}^{R_{4}-x} E_{s} \kappa y^{2} \sqrt{R_{4}^{2}-(x+y)^{2}} d y\right.
\end{aligned}
$$

$$
\begin{aligned}
& +\int_{0}^{R_{3}-x} E_{s} \kappa y^{2}\left[\sqrt{R_{4}^{2}-(x+y)^{2}}-\sqrt{R_{3}^{2}-(x+y)^{2}}\right] d y \\
& +\int_{R_{2}-x}^{R_{3}-x} E_{c} \kappa y^{2} \sqrt{R_{3}^{2}-(x+y)^{2}} d y \\
& +\int_{0}^{R_{2}-x} E_{c} \kappa y^{2}\left[\sqrt{R_{3}^{2}-(x+y)^{2}}-\sqrt{R_{2}^{2}-(x+y)^{2}}\right] d y \\
& +\int_{R_{1}-x}^{R_{2}-x} E_{s} \kappa y^{2} \sqrt{R_{2}^{2}-(x+y)^{2}} d y \\
& +\int_{0}^{R_{1}-x} E_{s} \kappa y^{2}\left[\sqrt{R_{2}^{2}-(x+y)^{2}}-\sqrt{R_{1}^{2}-(x+y)^{2}}\right] d y \\
& +\int_{0}^{-x} E_{s} \kappa y^{2}\left[\sqrt{R_{4}^{2}-(x+y)^{2}}-\sqrt{R_{3}^{2}-(x+y)^{2}}\right] d y \\
& +\int_{-x}^{-R_{3}-x} E_{s} \kappa y^{2}\left[\sqrt{R_{4}^{2}-(x+y)^{2}}-\sqrt{R_{3}^{2}-(x+y)^{2}}\right] d y \\
& +\int_{-R_{3}-x}^{-R_{4}-x} E_{s} \kappa y^{2} \sqrt{R_{4}^{2}-(x+y)^{2}} d y \\
& +\int_{0}^{-x} E_{s} \kappa y^{2}\left[\sqrt{R_{2}^{2}-(x+y)^{2}}-\sqrt{R_{1}^{2}-(x+y)^{2}}\right] d y \\
& +\int_{-x}^{-R_{1}-x} E_{s} \kappa y^{2}\left[\sqrt{R_{2}^{2}-(x+y)^{2}}-\sqrt{R_{1}^{2}-(x+y)^{2}}\right] d y \\
& \left.+\int_{-R_{1}-x}^{-R_{2}-x} E_{s} \kappa y^{2} \sqrt{R_{2}^{2}-(x+y)^{2}} d y\right\} \\
& \mathrm{M}=\overline{E I} \kappa
\end{aligned}
$$

Where:

$$
\begin{aligned}
\sigma_{x}= & \text { The normal stress } \\
\mathrm{A}= & \text { The integrated stressed area } \\
\kappa= & \text { The curvature } \\
\mathrm{x}= & \text { The neutral axis position measured from the } \\
& \text { center of the section } \\
\mathrm{y}= & \text { The distance of the examined fiber from the } \\
& \text { neutral axis }
\end{aligned}
$$

Finally, the elastic resistance moment can be determined, as the one that causes the section's most critical fiber to yield. Candidates for critical fiber are (i) the most distant from the neutral axis point of the outer steel face, in the area under tension where concrete remains inactive, or (ii) the most distant from the neutral axis point of the concrete core, in the compressed zone, where this-significantly weaker than steel-material is stressed the most. Thus, Equation 8 gives the elastic resistance moment:

$\mathrm{M}_{e l}=\min \left\{\mathrm{M}_{s, \max }=\frac{f_{s y} \overline{E I}}{E_{s}\left(R_{4}+x\right)}, \mathrm{M}_{c, \max }=\frac{f_{c} \overline{E I}}{E_{c}\left(R_{3}-x\right)}\right\}$ 
Where:

$M_{e l} \quad=$ The elastic resistance moment

$M_{s, \max }=$ The maximum moment corresponding to first yielding of steel

$M_{c, \max }=$ The maximum moment corresponding to first yielding of concrete

Beyond the elastic region, different scenarios have to be considered and checked, regarding the expansion of yielding over the cross section. The derived expressions in this case are, apparently, far more complex and are not presented here. Nevertheless, of practical importance is the plastic resistance moment of the section. From Equation 9 and 10, assuming that the whole section is plastified, the plastic neutral axis position and the plastic resistance moment $M_{p l}$ can be easily calculated, respectively:

$$
\begin{aligned}
& \int_{\mathrm{A}} \sigma_{x, p l} d A=0 \Rightarrow \\
& \int_{R_{3}-x_{p l}}^{R_{4}-x_{p l}} f_{s y} \sqrt{R_{4}^{2}-\left(x_{p l}+y\right)^{2}} d y+ \\
& \int_{0}^{R_{3}-x_{p l}} f_{s y}\left[\sqrt{R_{4}^{2}-\left(x_{p l}+y\right)^{2}}-\sqrt{R_{3}^{2}-\left(x_{p l}+y\right)^{2}}\right] d y+ \\
& \int_{R_{2}-x_{p l}}^{R_{3}-x_{p l}} f_{c} \sqrt{R_{3}^{2}-\left(x_{p l}+y\right)^{2}} d y+ \\
& \int_{0}^{R_{2}-x_{p l}} f_{c}\left[\sqrt{R_{3}^{2}-\left(x_{p l}+y\right)^{2}}-\sqrt{R_{2}^{2}-\left(x_{p l}+y\right)^{2}}\right] d y+ \\
& \int_{R_{1}-x_{p l}}^{R_{2}-x_{p l}} f_{s y} \sqrt{R_{2}^{2}-\left(x_{p l}+y\right)^{2}} d y+ \\
& \int_{0}^{R_{1}-x_{p l}} f_{s y}\left[\sqrt{R_{2}^{2}-\left(x_{p l}+y\right)^{2}}-\sqrt{R_{1}^{2}-\left(x_{p l}+y\right)^{2}}\right] d y+ \\
& \int_{0}^{-x_{p l}} f_{s y}\left[\sqrt{R_{4}^{2}-\left(x_{p l}+y\right)^{2}}-\sqrt{R_{3}^{2}-\left(x_{p l}+y\right)^{2}}\right] d y+ \\
& \int_{-x_{p l}}^{-\left(R_{3}-x_{p l}\right)} f_{s y}\left[\sqrt{R_{4}^{2}-\left(x_{p l}+y\right)^{2}}-\sqrt{R_{3}^{2}-\left(x_{p l}+y\right)^{2}}\right] d y+ \\
& \int_{-\left(R_{3}+x_{p l}\right)}^{-\left(R_{4}+x_{p l}\right)} f_{s y} \sqrt{R_{4}^{2}-\left(x_{p l}+y\right)^{2}} d y+ \\
& \int_{0}^{-x_{p l}} f_{s y}\left[\sqrt{R_{2}^{2}-\left(x_{p l}+y\right)^{2}}-\sqrt{R_{1}^{2}-\left(x_{p l}+y\right)^{2}}\right] d y+ \\
& \int_{-x_{p l}}^{-\left(R_{1}+x_{p l}\right)} f_{s y}\left[\sqrt{R_{2}^{2}-\left(x_{p l}+y\right)^{2}}-\sqrt{R_{1}^{2}-\left(x_{p l}+y\right)^{2}}\right] d y+ \\
& \int_{-\left(R_{1}+x_{p l}\right)}^{-\left(R_{2}+x_{p l}\right)} f_{s y} \sqrt{R_{2}^{2}-\left(x_{p l}+y\right)^{2}} d y=0 \\
& M_{p l}=2\left\{\int_{R_{3}-x_{p l}}^{R_{4}-x_{p l}} f_{s y} y \sqrt{R_{4}^{2}-\left(x_{p l}+y\right)^{2}} d y+\right.
\end{aligned}
$$

$$
\begin{aligned}
& \int_{0}^{R_{3}-x_{p l}} f_{s y} y\left[\sqrt{R_{4}^{2}-\left(x_{p l}+y\right)^{2}}-\sqrt{R_{3}^{2}-\left(x_{p l}+y\right)^{2}}\right] d y+ \\
& \int_{R_{2}-x_{p l}}^{R_{3}-x_{p l}} f_{c} y \sqrt{R_{3}^{2}-\left(x_{p l}+y\right)^{2}} d y+ \\
& \int_{0}^{R_{2}-x_{p l}} f_{c} y\left[\sqrt{R_{3}^{2}-\left(x_{p l}+y\right)^{2}}-\sqrt{R_{2}^{2}-\left(x_{p l}+y\right)^{2}}\right] d y+ \\
& \int_{R_{1}-x_{p l}}^{R_{2}-x_{p l}} f_{s y} y \sqrt{R_{2}^{2}-\left(x_{p l}+y\right)^{2}} d y+ \\
& \int_{0}^{R_{1}-x_{p l}} f_{s y} y\left[\sqrt{R_{2}^{2}-\left(x_{p l}+y\right)^{2}}-\sqrt{R_{1}^{2}-\left(x_{p l}+y\right)^{2}}\right] d y+ \\
& \int_{0}^{-x_{p l}} f_{s y} y\left[\sqrt{R_{4}^{2}-\left(x_{p l}+y\right)^{2}}-\sqrt{R_{3}^{2}-\left(x_{p l}+y\right)^{2}}\right] d y+ \\
& \int_{-x_{p l}}^{-\left(R_{3}-x_{p l}\right)} f_{s y} y\left[\sqrt{R_{4}^{2}-\left(x_{p l}+y\right)^{2}}-\sqrt{R_{3}^{2}-\left(x_{p l}+y\right)^{2}}\right] d y+ \\
& \int_{-\left(R_{3}+x_{p l}\right)}^{-\left(R_{4}+x_{p l}\right)} f_{s y} y \sqrt{R_{4}^{2}-\left(x_{p l}+y\right)^{2}} d y+ \\
& \int_{0}^{-x_{p l}} f_{s y} y\left[\sqrt{R_{2}^{2}-\left(x_{p l}+y\right)^{2}}-\sqrt{R_{1}^{2}-\left(x_{p l}+y\right)^{2}}\right] d y+ \\
& \int_{-x_{p l}}^{-\left(R_{1}+x_{p l}\right)} f_{s y} y\left[\sqrt{R_{2}^{2}-\left(x_{p l}+y\right)^{2}}-\sqrt{R_{1}^{2}-\left(x_{p l}+y\right)^{2}}\right] d y+ \\
& \left.\int_{-\left(R_{1}+x_{p l}\right)}^{-\left(R_{2}+x_{p l}\right)} f_{s y} y \sqrt{R_{2}^{2}-\left(x_{p l}+y\right)^{2}} d y\right\}
\end{aligned}
$$

\section{Analytical Procedure Verification}

The above expressions, describing the elastic and plastic behavior of a sandwich tubular section under axial load or bending moment, were coded in Mathematica software (Wolfram Research Inc., 2012), for further manipulation and parametrical analyses, in order for qualitative conclusions to be extracted. The verification of the developed algorithm's correctness was carried out by means of comparison of its results with those obtained from Biaxial Bending software (Charalampakis and Koumousis, 2008), specialized in the analysis of composite sections under axial and bending loads and those extracted from the FE analysis software Adina (ADINA R\&D Inc., 2006).

The model incorporating the sandwich-type section in the Adina environment consists of a cylinder, fixed at its bottom section and subjected to pure bending by a concentrated moment exerted on it at its top section. The cylinder is modeled with 3D-Solid elements representing the concrete core and shell elements simulating the two steel faces. The 3D-Solid elements shared the same nodes, with each shell-elements face, so that full bond is assumed to remain intact throughout the analyses. A part of the model is presented in Fig. 5a. 
In addition to the extracted moment-rotation valuepairs which describe the model's behavior, the determination of the neutral axis position is also possible using the contour stress results of any section along the tower model, as shown in Fig. 5b. In all cases, the determined position was the same as the one calculated by the analytical solution.

The results obtained from the analysis carried out in Biaxial Bending, for numerous combinations of geometries, loads and material properties, perfectly matched those derived from the analytical approach and those of the Adina simulation. The comparison was made in terms of elastic resistance moment, plastic bending strength and initial stiffness. The comparative moment-curvature chart in Fig. 6 demonstrates the coincidence of the results between the three different approaches, indicatively for $E_{s}=210 \mathrm{GPa}, f_{s y}=355$ $\mathrm{MPa}, E_{c}=32 \mathrm{GPa}, f_{c}=30 \mathrm{MPa}, t_{s}=10 \mathrm{~mm}, t_{c}=100$ $\mathrm{mm}$ and $R_{4}=2000 \mathrm{~mm}$.

Concerning the determination of plastic bending strength, it should be noted that concrete was assumed capable of following steel up to its ultimate strain, while still maintaining its maximum strength. This assumption is a means to avoid a significant amount of complexity in the computations of the analytical approach, but in some cases it introduces a computational error. The latter is qualitatively presented in Fig. 7, in comparison to a more realistic consideration of a concrete ultimate strain equal to $3.5 \%$. Extensive testing in Biaxial Bending showed, however, that the aforementioned error in plastic bending strength calculation does not exceed 5\%, irrespective of layers' thicknesses, as long as the steel-toconcrete strength ratio remains less than $18\left(f_{s} / f_{c}<18\right)$.

\section{Parametric Analysis and Optimization}

Following the verification of the expressions presented in the previous section, extensive parametric analyses were performed via the developed Mathematica code, in order to investigate the influence of different combinations of key-factors on the section's flexural stiffness and strength. Assuming a sandwich section with an external diameter of $4 \mathrm{~m}\left(R_{4}=\right.$ $2 \mathrm{~m})$, key-factors are the thicknesses of core and faces regarding the geometry of the section and yield strength and Young's modulus of steel and concrete, as far as material properties are concerned. It should be mentioned that, typically, the design of wind turbine towers, especially those of large size and consequently high construction costs, is too conservative for any post-yield reserves to be taken into account. The investigation presented in this chapter is, thus, limited within the section's elastic range.

Retaining the diameter constant at $4 \mathrm{~m}$, a steel face thickness was firstly selected, along with the properties of the materials incorporated in the sandwich section. The core thickness was then introduced into the algorithm, taking increasing values within a range from $t_{c}=10 \mathrm{~mm}$ to $t_{c}=400 \mathrm{~mm}$ with a step of $10 \mathrm{~mm}$ and the behavior of both the elastic bending strength and initial stiffness was recorded, as a function of concrete to steel thicknesses ratio. In Fig. 8, the result of such a numerical investigation is presented, for steel face thickness $t_{s}=5 \mathrm{~mm}$, steel yield-strength $f_{s y}=355 \mathrm{MPa}$, steel Young's modulus $E_{s}=210 \mathrm{GPa}$, concrete strength $f_{c}=30 \mathrm{MPa}$ and concrete Young's modulus $E_{c}=32 \mathrm{GPa}$.

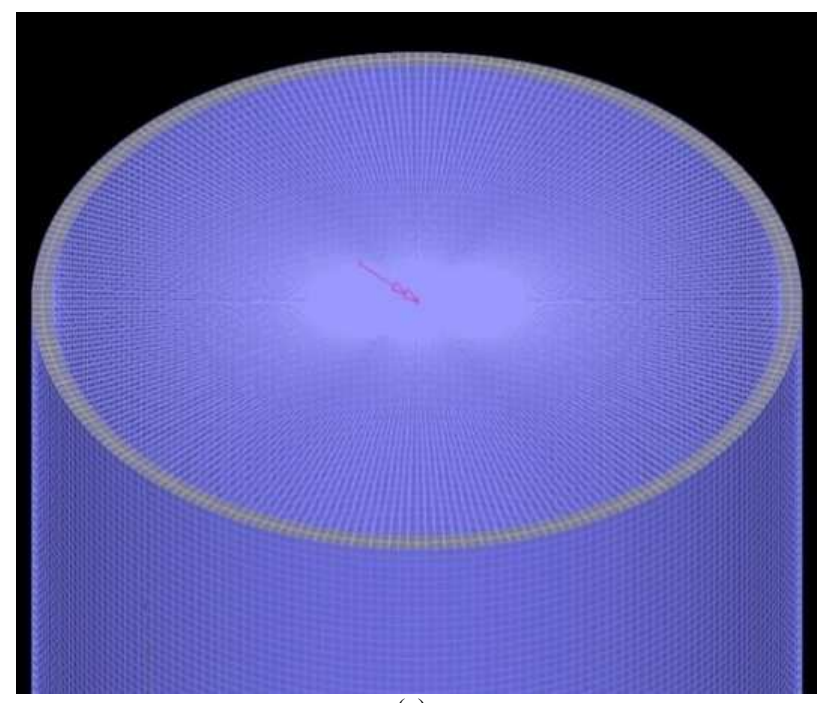

(a)

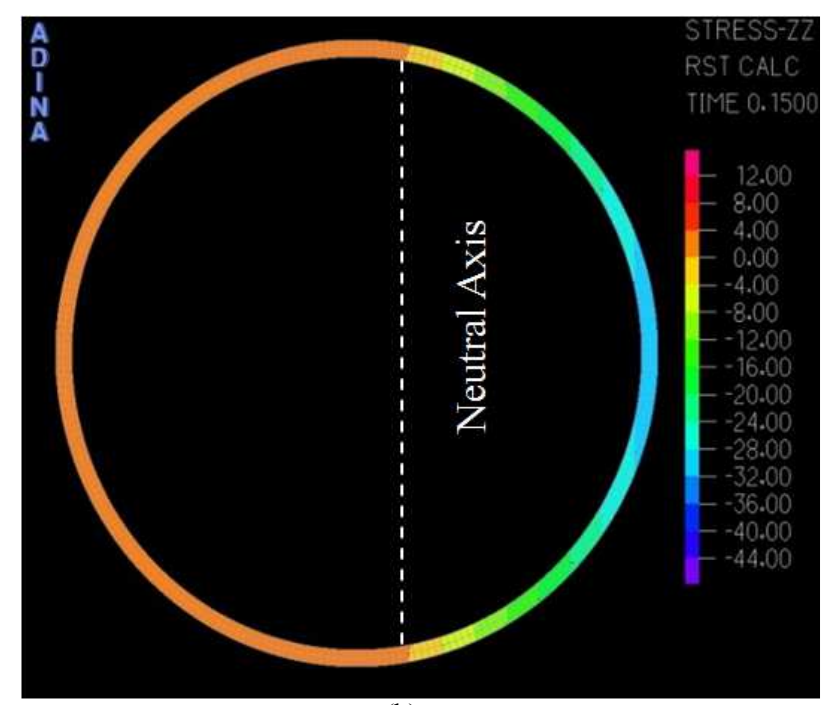

(b)

Fig. 5. (a) Sandwich-section cylindrical finite-element model in Adina (b) neutral-axis determination through contour stress results 


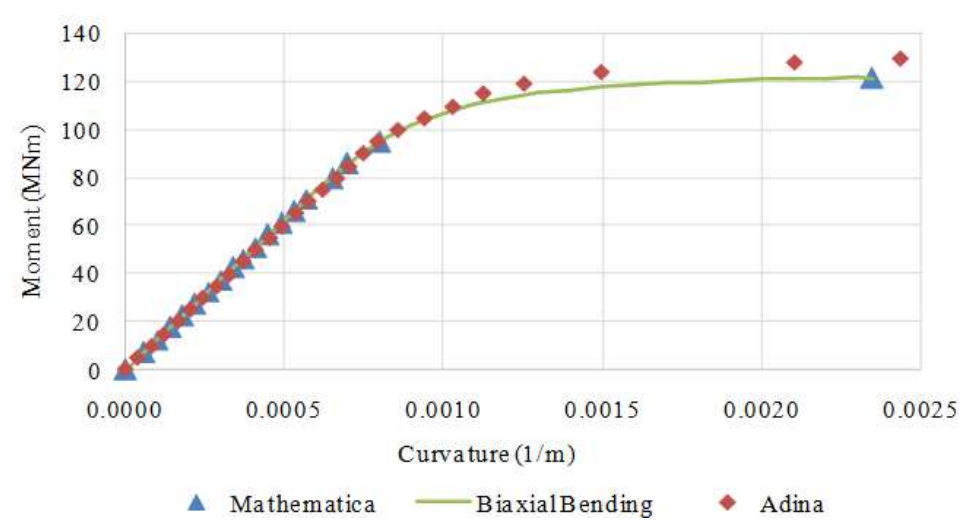

Fig. 6. Comparison of moment-curvature results between the analytical solution, Biaxial Bending and Adina, for a sandwich-type section of $E_{s}=210 \mathrm{GPa}, f_{s y}=355 \mathrm{MPa}, E_{c}=32 \mathrm{GPa}, f_{c}=30 \mathrm{MPa}, t_{s}=10 \mathrm{~mm}, t_{c}=100 \mathrm{~mm}, R_{4}=2000 \mathrm{~mm}$

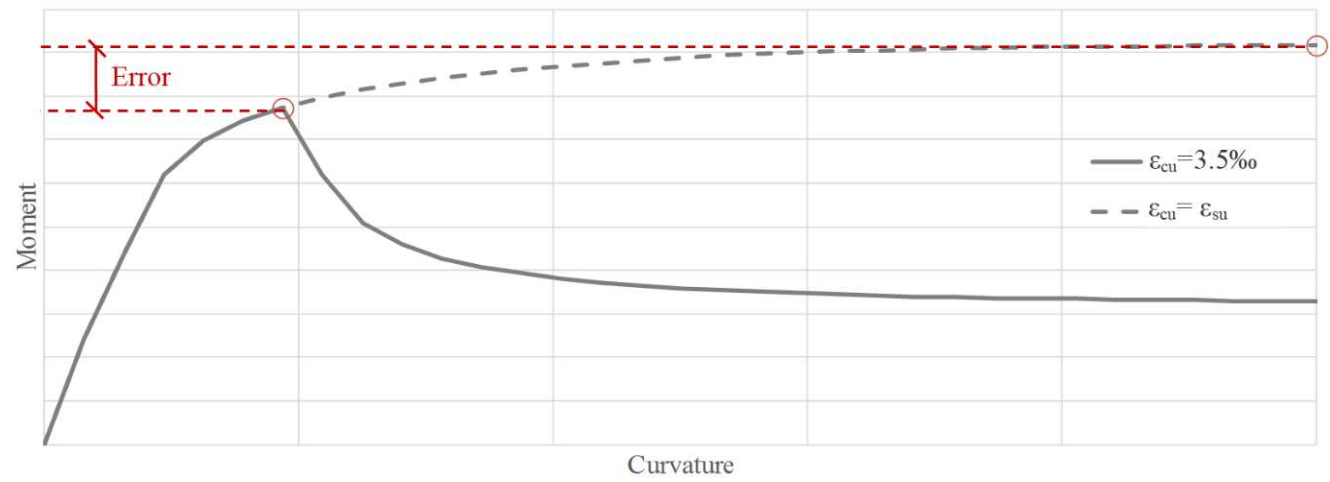

Fig. 7. Qualitative depiction of error due to prolongation of concrete ultimate strain
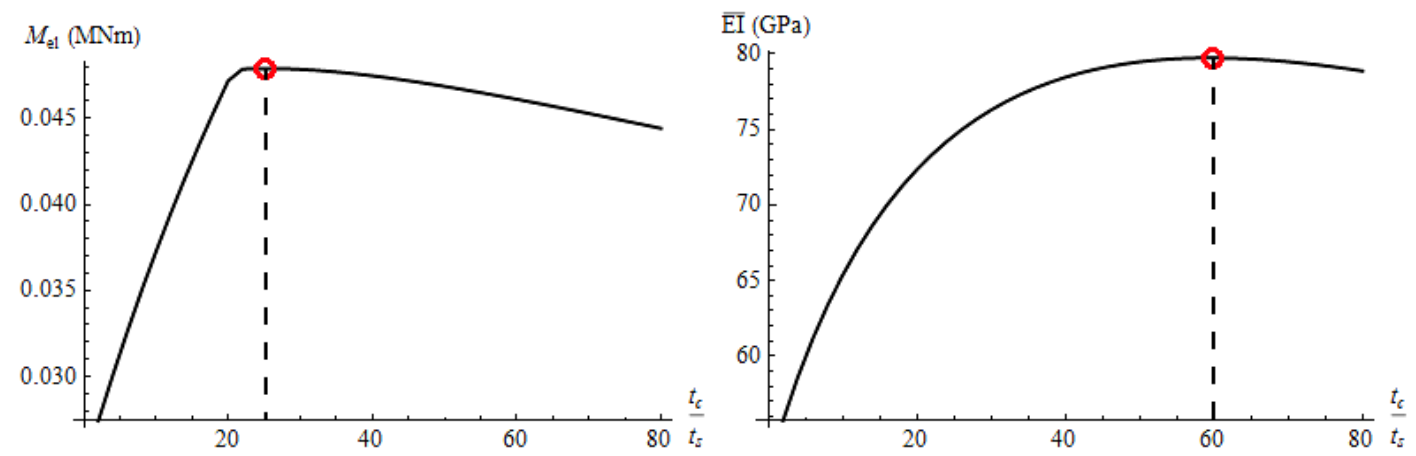

Fig. 8.Influence of concrete and steel thicknesses on the elastic bending strength and the initial stiffness of a sandwich-type section with the following properties: $E_{s}=210 \mathrm{GPa}, f_{s y}=355 \mathrm{MPa}, E_{c}=32 \mathrm{GPa}, f_{c}=30 \mathrm{MPa}, t_{s}=5 \mathrm{~mm}, R_{4}=2000 \mathrm{~mm}$

The qualitative conclusion inferred from Fig. 8 and several similar charts resulting for different face thicknesses and material properties, is that, in all cases, there are two specific core thickness values maximizing the elastic bending strength or the initial stiffness of the sandwich section, respectively. As for Fig. 8 in particular, these two values are $25 \mathrm{~mm}$ and $60 \mathrm{~mm}$, respectively, the latter being more than twice the former.

A twofold need arises from this observation. On the one hand is that of determining those two core thickness values, which maximize each of the two factors in question, namely the elastic bending strength and initial stiffness. On the other hand is the need of seeking that third core-thickness value, which sufficiently bridges the gap between the two previous values and, thus, satisfies both criteria as decently as possible.

The results of these two endeavors are demonstrated in Fig. 9, indicatively for two combinations of material properties. More specifically, in each case, for every steel face thickness appearing on the horizontal axis, the left and right bars represent the core thickness maximizing the elastic bending strength and the initial 
stiffness, respectively. The pertinent maximum values achieved also appear over each thickness pair. Finally, the difference in percentage between each two optimal core thicknesses is indicated, so that the minimum of differences can be determined. As a total, 15 combinations were similarly examined, consisting of
3 steel and 5 concrete types $\left(\left\{E_{s}=210 \mathrm{GPa}, f_{s y}=235\right.\right.$ $\mathrm{MPa}\},\left\{E_{s}=210 \mathrm{GPa}, f_{s y}=355 \mathrm{MPa}\right\},\left\{E_{s}=210 \mathrm{GPa}\right.$, $\left.f_{s y}=460 \mathrm{MPa}\right\}$ and $\left\{E_{c}=29 \mathrm{GPa}, f_{c}=20 \mathrm{MPa}\right\},\left\{E_{c}=\right.$ $\left.30.5 \mathrm{GPa}, f_{c}=25 \mathrm{MPa}\right\},\left\{E_{c}=32 \mathrm{GPa}, f_{c}=30 \mathrm{MPa}\right\}$, $\left\{E_{c}=33.5 \mathrm{GPa}, f_{c}=35 \mathrm{MPa}\right\},\left\{E_{c}=35 \mathrm{GPa}, f_{c}=40\right.$ $\mathrm{MPa}\})$.
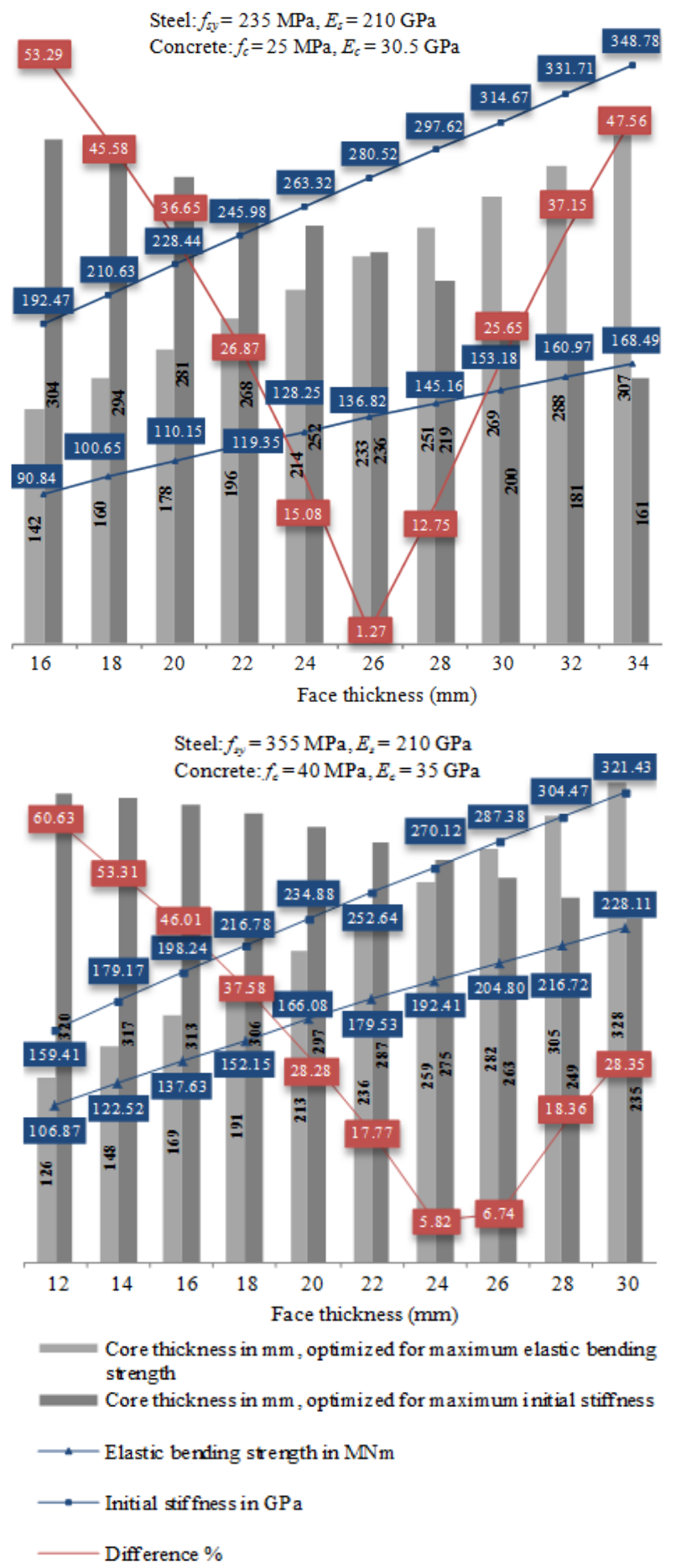

Fig. 9. Optimal combinations of concrete-core and steel-faces thicknesses, for maximization of elastic moment resistance and stiffness, for $R_{4}=2000 \mathrm{~mm}$ 


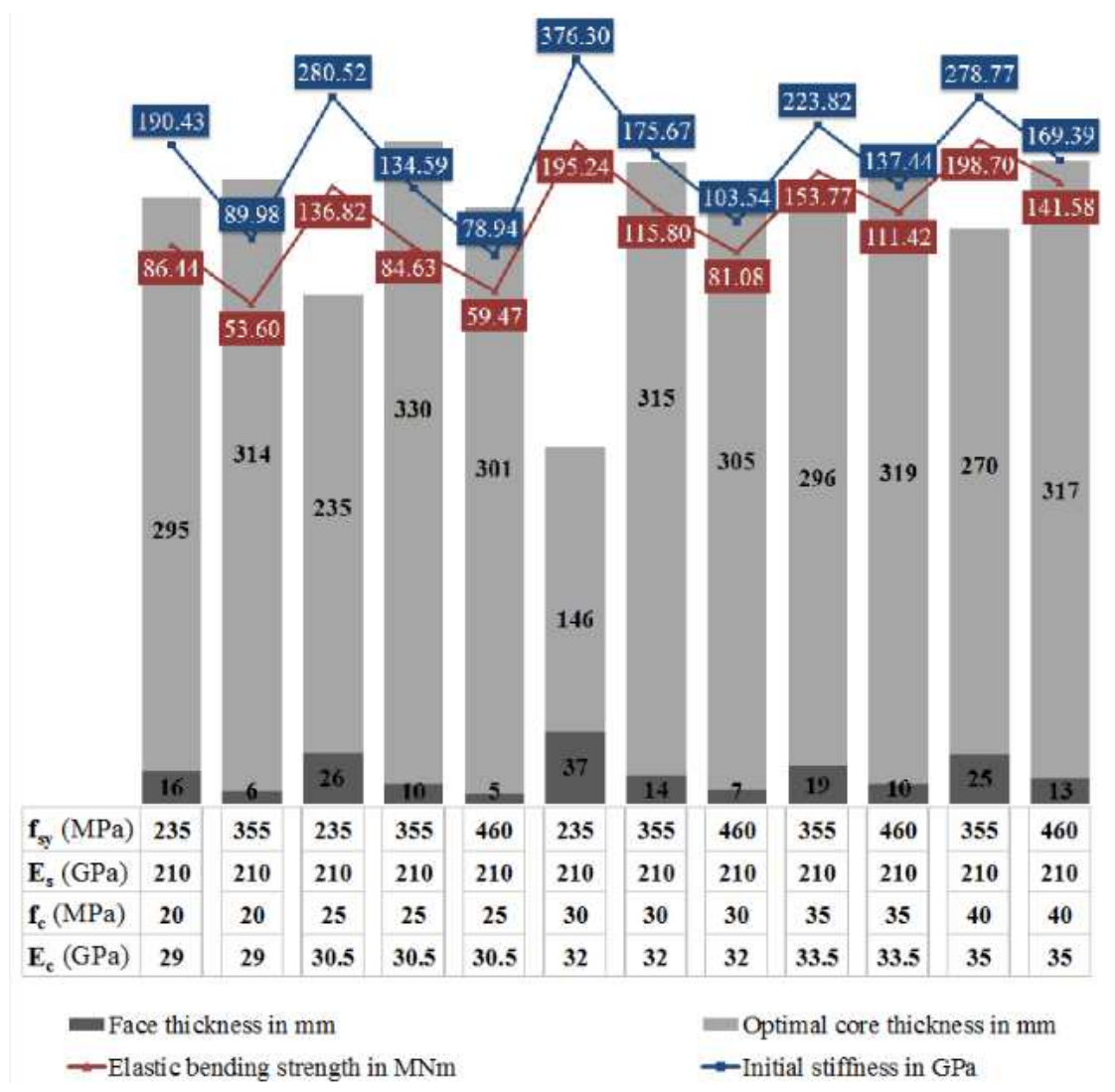

Fig. 10. Concrete-core and steel-faces thickness combinations providing convergence of the criteria of elastic moment resistance and stiffness maximization, for an outer radius of $R_{4}=2000 \mathrm{~mm}$ and various combinations of material properties

The above material combinations and the corresponding optimal face and core thicknesses, leading to the convergence of the two criteria, are summarized in Fig. 10, along with the resulting values of elastic bending strength and initial stiffness. The absence of some combinations is due to their inability to adequately fulfill both criteria, regardless of section's geometry.

Hence, Fig. 10 provides a means of determining the optimal combination of geometry and material qualities of a sandwich section as far as its static behavior in bending is concerned, according to any specific requirements, ranging from approximately 53 to 199 $\mathrm{MNm}$ of elastic resistance moment and between 79 to $376 \mathrm{GPa}$ of elastic stiffness. Thus, improper selections of material thicknesses and qualities providing the required strength by sacrificing stiffness and vice versa can be avoided, as can be any other options satisfying both criteria but implying redundant material quantities, against structural economy.

\section{Conclusion}

The option of a sandwich type section consisting of two steel faces and a concrete core was investigated at preliminary design level, as an alternative for large wind turbine towers, of such heights that the conventional steel tubular sections become unfeasible, due to both transportation incapability and structural economy. The analytical expressions describing the section's behavior under axial loading or pure bending were formulated up to the yield point, which, as a limit, governs the design of wind turbine towers, as well as up to the ultimate point. The expressions were verified by comparison of the resulting moment-curvature diagrams with those obtained from two programs, namely Biaxial Bending and Adina. A pertinent Mathematica code was subsequently developed and utilized for an extensive parametric analysis, involving a wide range of material properties and thicknesses of section's core and faces. For each selection of face thicknesses and material qualities, the thickness of the core maximizing the elastic resistance moment was determined, along with that leading to a maximum initial stiffness. In each case, finally, the determination of the optimal core thickness, which provides the most efficient solution regarding both criteria, was addressed. The resulting, summarizing chart provides a means of identifying both proper and inefficient or uneconomical solutions, as an early-stage design tool for sandwich-type, wind turbine tower sections. 
It should be mentioned that, besides the evident efficiency-gain regarding the static behavior of a wind turbine tower, the effects of the above optimized sandwich sections on the tower's dynamic response cannot be omitted, considering the eminently dynamic nature of wind as the main loading source of a wind turbine. More specifically, it is highly possible that the considerable amount of additional mass, involved in the sandwich solution, might lead to an undesirable dynamic behavior of the structure. Indeed, preliminary dynamic analyses, performed as a successive phase of the current research, tend to verify this presumption, thus rendering a dynamic-response-based optimization an endeavor of significant practical value. From a design point of view this would imply to propose sandwich-type sections for the lower part of the tower and steel-only tubular sections for the upper part, in order to avoid the undesirable effects of increased mass at large height.

\section{Acknowledgement}

The authors would like to thank Dr. Aristotelis Charalampakis for his help with software Biaxial Bending.

\section{Funding Information}

This research has been co-financed by the European Union (European Social Fund-ESF) and Hellenic National Funds through the Operational Program "Education and Lifelong Learning" (NSRF 2007-2013) Research Funding Program “Aristeia II”.

\section{Authors' Contributions}

The two authors have participated equally in the research work.

\section{Ethics}

There are no ethical issues known to authors, that may arise after the publication of this manuscript.

\section{References}

ADINA R\&D Inc. 2006. Theory and Modeling GuideVolume I: ADINA, Report ARD 08-7.

Banhart, J., 2001. Manufacture, characterisation and application of cellular metals and metal foams. Progress Mater. Sci., 46: 559-632.

DOI: $10.1016 / \mathrm{S} 0079-6425(00) 00002-5$

Basu, B., 2010. Tower Design and Analysis. In: Wind Power Generation and Wind Turbine Design, Tong, W. (Ed.), WIT Press, Southampton, UK, ISBN-13: 978-1-84564-205-1, pp: 527-557.
Burton, T., D. Sharpe, N. Jenkins and E. Bossanyi, 2001. Wind Energy Handbook. 1st Edn., John Wiley and Sons, New York, ISBN-10: 0471489972, pp: 642.

Charalampakis, A. and V. Koumousis, 2008. Ultimate strength analysis of composite sections under biaxial bending and axial load. Adv. Eng. Software, 39: 923-936. DOI: 10.1016/j.advengsoft.2008.01.007

DNV, 2002. Guidelines for Design of Wind Turbines. 2nd Edn., DNV/Risø, Copenhagen, Denmark. ISBN-10: 87-550-2870-5, pp: 286.

Hau, E., 2006. The Tower. In: Wind Turbines, Springer, Berlin, Germany, ISBN: 978-3-540-24240-6, pp: 421-444.

Lim, S., C. Kong and H. Park, 2013. A study on optimal design of filament winding composite tower for 2 MW class horizontal axis wind turbine systems. Int. J. Composite Mater., 3: 15-23.

NREL, 2014. Analysis of transportation and logistics challenges affecting the deployment of larger wind turbines: Summary of results. National Renewable Energy Laboratory, Colorado, USA.

Schaumann, P. and C. Keindorf, 2008. Sandwichtowers for wind energy converters. DEWI Magazin, 33: 65-76.

Simančík, F., 2001. Metallic foams-ultra light materials for structural applications. Inźynieria Materiałowa, 5: 823-828.

Twele, J., C. Heilmann and M. Schubert, 2012. Design and Components. In: Wind Power Plants, Gasch, R. and J. Twele (Eds.), Springer, Heidelberg, Germany, ISBN: 978-3-642-22937-4, pp: 94-101.

Vernardos, S. and C. Gantes, 2014. Preliminary feasibility investigation of sandwich type shells for wind turbine towers. Proceedings of the 8th Hellenic National Conference on Steel Structures, Oct. 2-4, M.S.R.S., Greece.

Wolfram Research Inc., 2012. Mathematica. Illinois, USA. 